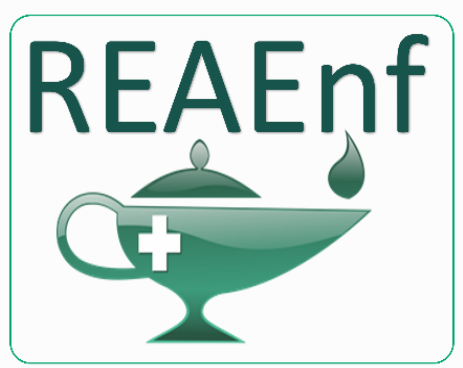

Revista Eletrônica Acervo Enfermagem
REVISÃO BIBLIOGRÁFICA

Recebido em: 8/2020

Aceito em: 9/2020

Publicado em: 11/2020

\title{
Humanização da assistência de enfermagem na classificação de risco nos serviços de urgência e emergência
}

\author{
Humanization of nursing assistance in risk classification in emergency and emergency
}

Humanización de la asistencia de enfermería en la clasificación de riesgos en servicios de emergencia y de emergencia

Rayanne Lúcia de Oliveira Campos ${ }^{1 *}$, Niedja Carla Dias de Lira e Silva ${ }^{2}$, Adrian Thaís Cardoso Santos Gomes da Silva ${ }^{1}$, Manoela Rodrigues de Santana ${ }^{3}$, Luany Abade Café ${ }^{1}$, Luan Naís de Souza $^{4}$, Ada Evellyn Galdino da Silva ${ }^{5}$, Edivania Cristina da Silva ${ }^{6}$, Amanda Domingos da Silva ${ }^{7}$.

Resumo: Este artigo buscou compreender a humanização da assistência de enfermagem na classificação de risco nos serviços de urgência e emergência. Para a realização desse estudo foi realizado um levantamento bibliográfico nas bases de dados da Scientific Eletronic Library Online (SCIELO), Literatura Científica e Técnica da América Latina e Caribe (LILACS), Medical Literature Analysis and Retrieval System Online (MEDLINE) no período de janeiro de 2020. O acolhimento com classificação de risco é uma ferramenta importante dentro dos serviços de urgência e emergência que possuem como finalidade organizá-los de forma a prática da escuta qualificada e priorizar a classificação de casos através da sua gravidade. Além dos conhecimentos técnicos e científicos a enfermagem necessita praticar uma assistência baseada na humanização que pode ser alcançada através do atendimento e acolhimento, assim considerando o indivíduo como um ser holístico, buscar não só enxergar a doença, mas, atender ao doente de acordo com suas necessidades.

Palavras-chave: Diagnóstico, Educação em enfermagem, Humanização da assistência.

\begin{abstract}
This article sought to understand the humanization of nursing care in the classification of risk in urgent and emergency services. In order to carry out this study, a bibliographical survey was carried out in the databases of the Scientific Eletronic Library Online (SCIELO), Scientific and Technical Literature of Latin America and the Caribbean (LILACS), Medical Literature Analysis and Retrieval System Online (MEDLINE) in January 2020. The reception with risk classification is an important tool within the urgency and emergency services that aim to organize them in order to practice qualified listening and prioritize the classification of cases according to their severity. In addition to technical and scientific knowledge, nursing needs to practice assistance based on humanization that can be achieved through care and reception, thus considering the individual as a holistic being, seeking not only to see the disease but to attend to the patient according to their needs.
\end{abstract}

Keywords: Diagnosis, Nursing education, Humanization of assistance.

\footnotetext{
1 Universidade Federal de Pernambuco. Recife - PE. *E-mail: rayanne_lucia95@hotmail.com

2 Centro Universitário São Miguel. Recife - PE.

${ }^{3}$ Universidade Católica de Pernambuco. Recife - PE.

${ }^{4}$ Faculdade Pernambucana de Saúde. Recife - PE.

${ }^{5}$ Centro universitário Estácio do Recife. Recife - PE.

${ }^{6}$ Centro Universitário dos Guararapes. Jaboatão dos Guararapes - PE.

${ }^{7}$ Centro de Ensino e Pesquisa em Emergências Médicas (CEPEM). Recife - PE.
} 
Resumen: Este artículo buscaba comprender la humanización de los cuidados de enfermería en la clasificación de riesgos en los servicios de urgencia y emergencia. Para la realización de este estudio, se realizó un relevamiento bibliográfico en las bases de datos de la Biblioteca Científica Electrónica en Línea (SCIELO), Literatura Científica y Técnica de América Latina y el Caribe (LILACS), Sistema de Análisis y Recuperación de Literatura Médica en Línea (MEDLINE) en enero 2020. La recepción con clasificación de riesgo es una herramienta importante dentro de los servicios de urgencia y emergencia que tienen como objetivo organizarlos para practicar la escucha calificada y priorizar la clasificación de los casos según su gravedad. Además de los conocimientos técnicos y científicos, la enfermería necesita practicar la asistencia basada en la humanización que se puede lograr a través del cuidado y la recepción, considerando así al individuo como un ser holístico, buscando no solo ver la enfermedad sino atender al paciente de acuerdo a sus necesidades.

Palabras clave: Diagnóstico, Educación en enfermería, Humanización de la Asistencia.

\section{INTRODUÇÃO}

A transição demográfica, social e epidemiológica faz com que a procura e a oferta dos serviços de saúde no Brasil seja um fenômeno que sofre alterações constantes. Nesse sentido, nos últimos anos tem-se observado o aumento da demanda no atendimento de urgências e emergências (OLIVEIRA JLC, et al., 2017).

A classificação de risco é um processo dinâmico usado como base conceitos e escalas internacionais. Os riscos são classificados em 5 níveis, apresentando uma maior confiabilidade na avaliação. Dentre os modelos de escalas destacam-se: a escala australiana: Australasian Triage Scale (ATS); o protocolo canadense Canadian Triage Acuity Scale (CTASO); a escala norte-americana: Emergency Severity Index (ESI), e o protocolo inglês: Manchester Triage System (protocolo de Manchester) (PAGLIOTO LF, et al., 2016).

A classificação de risco tem como objetivo organizar de forma mais objetiva o atendimento de acordo com a gravidade do paciente, e não por ordem de chegada, com isso o paciente diminui o risco, o tempo de espera, e garante o encaminhamento para os outros serviços de saúde se necessário. Essa nova forma de atendimento funciona para cumprir as diretrizes do Sistema Único de Saúde (SUS) principalmente aquele que concerne à equidade, que se refere a dar mais para quem tem menos e dar menos para quem tem mais. Essa classificação é realizada através do protocolo clínico escolhido pela instituição previamente estabelecida e deve ser proposto pelo Ministério da Saúde (MS), devendo ser adaptado às necessidades de cada serviço de saúde (BARTEL TE, 2015).

As Unidade de Pronto Atendimento (UPAs), necessitam de um aporte governamental que engloba as três esferas de governo, que por sua vez desempenham competências e responsabilidades de acordo com sua responsabilidade federativa. As UPAs se configuram como uma porta de entrada dos usuários no SUS para atendimento referente às Urgências e Emergências, desse modo cenários de superlotação são frequentes e se relacionam com a quebra do sistema de comunicação e referenciamento que deveria partir da Atenção Básica, atenção especializada e hospitalar, devido à escassez de recursos humanos e materiais e a demanda variável de usuários que aumenta com a sazonalidade e pelo número de casos graves. (OLIVEIRA JLC, et al., 2017).

É proposto ao enfermeiro, a utilização de um fluxograma de atendimento para incentivá-lo a uma reflexão profunda sobre o processo de trabalho, assim o Ministério da Saúde designa para execução desta avaliação. Todo embasamento do enfermeiro é através de treinamento de acordo com os protocolos da unidade de saúde, realizando a classificação de risco, verificação dos sinais vitais, entrevista estruturada e em alguns casos a realização de exames complementares, desenvolve atividades como o acolhimento e a humanização durante a triagem (FREITAS FFB, et al., 2015).

De acordo com a resolução do COFEN no 423/2012 no âmbito da equipe de enfermagem, a classificação de risco e a priorização dos atendimentos nos serviços de urgência e emergência é privativa do enfermeiro, observando as disposições legais da profissão (COFEN, 2012). Porém, foi percebido que os atendimentos 
ofertados nos serviços de saúde eram realizados de maneira que apenas a doença era vista naquele paciente, sem considerar que o indivíduo é um ser biopsicossocial e necessita que o mesmo seja visto como um ser holístico e que seja acolhido com humanização, buscando compreender a realidade em eu que ele está inserido (SOUSA KHFJ, et al., 2018).

Dessa forma, o Ministério da Saúde (MS) criou em 2003 a Política Nacional de Humanização (PNH) com a finalidade de efetivar os princípios do SUS na rotina das práticas de atenção, gestão, e a qualificação pública do país. Devendo estar inserida em todas as outras políticas e nos programas do SUS. Além de proporcionar mudanças no processo de gestão da $\mathrm{PNH}$, buscam estimular a comunicação entre gestores, trabalhadores e usuários para que juntos possam construir o processo de forma coletiva no que tange às relações de poder, trabalho e afeto que muitas vezes combinam atitudes e práticas que são desumanizadoras inibindo a corresponsabilidade dos profissionais de saúde e dos usuários em seu cuidado (BRASIL, 2017).

Em relação às urgências e emergências, a PNH estabeleceu que esses serviços sofressem um processo de restauração através da implantação do Acolhimento com Classificação de Risco (ACCR). Nesse contexto, realiza-se uma avaliação inicial e procedem à seleção e encaminhamento do paciente as unidades adequadas de acordo com as necessidades. Destaca-se que a triagem é mais do que realizar a classificação dos pacientes em maior ou menor risco de vida se constitui como uma forma de garantir o direito à cidadania, acolher e orientar os indivíduos. Para que o processo seja simplificado utiliza-se de estratégias como os protocolos de classificação de risco que é realizado pelo enfermeiro para reduzir problemas (PAULA CFB, et al., 2019).

A atuação do enfermeiro na classificação de risco é de extrema importância pois, a escuta a queixa do usuário e tira todas as suas dúvidas. Desta forma, o enfermeiro cria uma relação de empatia com esse paciente reduzindo a ansiedade, agressividade ou impaciência que podem ocorrer na espera do atendimento (SOARES ACL, et al., 2018).

Dessa forma, o objetivo do presente estudo foi compreender a humanização da assistência de enfermagem na classificação de risco nos serviços de urgência e emergência.

\section{REVISÃO BIBLIOGRÁFICA}

\section{Humanização nos serviços de urgência e emergências}

Tem-se percebido cada vez mais no âmbito hospitalar a necessidade da humanização durante a assistência. Atualmente, é extremamente comum observar a fragmentação do ser humano dentro desses serviços que por diversas vezes o mesmo é compreendido apenas com as suas dimensões puramente biológicas. Essa situação decorre principalmente devido ao avanço da tecnologia no âmbito da saúde, que transformou cuidado na boa parte das vezes uma mera aplicação do procedimento técnico a fim de cumprir com o objetivo de cura (ASSIS LRS, et al., 2016).

Devido à superlotação nas unidades de urgência e emergência, a escassez de recursos a qualidade da assistência acaba sendo comprometida. Dessa forma, se configura como um problema de saúde pública e por consequência é necessário que a humanização seja integrada a assistência devido a essa realidade os que usuários enfrentam à procura desses serviços (PAULA CFB, et al., 2019). Para um cuidado humanizado é preciso uma equipe de saúde tenha responsabilidade, empoderamento científico, relação de troca de experiência com a equipe de saúde, para que possa desenvolver procedimentos para com o paciente, tendo como sujeito principal da assistência (ASSIS LRS, et al., 2016). Segundo o Humaniza SUS deve ser implantado o acolhimento com avaliação e classificação de risco nas unidades de urgência e emergência. Possibilitando que a demanda seja atendida e classificada de acordo com o seu quadro clínico, garantindo o acesso aos serviços de referências conforme necessário reduzindo o tempo na fila e de espera de atendimento (PRUDÊNCIO CPG, et al, 2016).

Nos estudos de Bartel TE, et al. (2015) contribuindo no cumprimento das diretrizes do SUS, em principal a equidade, no qual o atendimento é dar maior atenção a quem mais necessita, a forma de organizar o

REAEnf/EJNC | Vol. 5 | e5036 | DOI: https://doi.org/10.25248/REAenf.e5036.2020 Página 3 de 6 
atendimento com acolhimento com avaliação e classificação de risco, sendo um protocolo clínico proposto pelo MS e deve ser adaptado as necessidades de cada serviço, que deve ser avaliado o seu desempenho da estratégia no qual o acolhimento do usuário não é só parte do atendimento, faz parte administrativa e de encaminhamento de casos para serviços especializados.

Para que a humanização seja consolidada dentro dos serviços de urgência e emergência os usuários devem ser tratados como seres humanos integrados que possui sentimentos e particularidades para que se possa reduzir ansiedade dos mesmos. Os usuários que procuram atendimento nas diversas unidades encontram-se fragilizados e precisa que seja construída uma boa relação entre paciente e profissional onde as suas particularidades devem ser respeitadas (ANTUNES P, et al., 2018).

Porém, as condições de trabalho dentro do serviço de urgência e emergência são desgastantes e associada à falta de estrutura física são fatores que dificultam a oferta de um SUS humanizado. Dessa forma, acabam influenciando as relações do usuário com o serviço e por consequência desmotiva os profissionais e os indivíduos que procuram essas unidades de atendimento. Por isso se faz necessário que a humanização se expanda principalmente para os gestores de saúde, pois a partir do momento que se tem uma gestão que trabalha em prol de melhorar as condições de trabalho e recursos humanos, isso irá refletir de forma benéfica em quem está na linha de frente ao atendimento (MENEZES ML e SANTOS LRCS, 2017).

No que concerne à humanização dentro das unidades de urgência e emergência é dever das instituições realizar em planejamento e organização dos seus serviços com o objetivo de promover uma assistência de qualidade e que preserva a dignidade humana. Deve facilitar a realização do atendimento baseado na Constituição dos Direitos Humanos voltando a sua atenção para a prevenção de agravos e promoção das intervenções de acordo com as necessidades de cada paciente (SANTOS TTSM, 2017).

Segundo Antunes $P$, et al. (2018) queixas são frequentes pelos usuários do serviço de urgência e emergência, seja no atendimento público ou privado, que a assistência é focada na doença e não como o paciente como ser humano, por isto, a visão dos profissionais de saúde tem que ser holística em toda fase do atendimento, buscando o conforto e alívio no atendimento na urgência e emergência. Dentro das unidades de urgência e emergência deve ser realizado o acolhimento buscando demonstrar sensibilidade através dos profissionais de saúde que se constitui como ações importantes e decisivas para proporcionar aos pacientes e familiares à confiança e segurança necessárias para a assistência (ROCHA TRA e PINTO $F, 2016)$.

\section{Humanização na assistência de enfermagem na classificação de risco}

De acordo com a Portaria GM/MS no 2.048/2002 o atendimento aos casos de urgência e emergência deve iniciar por meio do acolhimento qualificado, garantindo uma assistência mais resolutiva. Bem como, enfatiza a necessidade da formação e qualificação dos profissionais envolvidos neste setor (NETO OC, et al., 2018).

O atendimento em urgência e emergência, sendo um atendimento rápido ao paciente, pois a prioridade é estabilizar o paciente, exigindo agilidade, habilidade e objetividade na prática de um enfermeiro, não observando a tensão e o medo diante daquela situação desconhecida, levando a uma fragilidade e por vezes agressividade como reação a insegurança que 0 atendimento gerou no paciente, a partir disso, 0 trabalho de enfermagem deve ser de forma sistemática, aplicando as intervenções inerentes a sua prática laboral, embasada em ações humanizadas, com estratégia individualizada de acordo com a necessidade de cada paciente (ANTUNES $P$, et al., 2018).

Para determinar a categoria de classificação o enfermeiro no acolhimento que tem a classificação de risco, toma como base na decisão, a escuta qualificada, o julgamento clínico e crítico das queixas que levam a um raciocínio lógico, no qual determinará o risco com o objetivo de compreender a informação clínica (FREITAS FFB, et al., 2015). Assim, o enfermeiro se apresenta como o profissional responsável por modificar a realidade do paciente. No âmbito do acolhimento busca ouvir suas queixas e possui como finalidade levantar as necessidades dos indivíduos desde físicas até sociais. A avaliação se inicia no 
momento da consulta de enfermagem na triagem, praticando a escuta qualificada, realizar anamnese e exame físico para decidir qual a conduta adequada para aquele indivíduo que procurou a unidade (PRUDÊNCIO CPG, et al., 2016).

Dessa forma, o enfermeiro para realizar a classificação de risco deve se basear na humanização e na sua prática diária através da escuta qualificada, pois, conseguem enxergar o indivíduo de forma que não busque conhecer apenas a patologia, mas como também o seu sofrimento, condições socioculturais, espirituais e econômicas no qual o indivíduo está inserido, pois é exatamente isto que torna o atendimento mais humano, e de certa forma está atrelado ao cuidado, que faz o sentido da profissão (FREITAS FFB, et al., 2015).

A humanização no âmbito do atendimento prestado pela enfermagem dentro do contexto da saúde possui um papel muito importante dentro contexto atual das unidades de saúde. Em uma assistência humanizada todo cuidado deve ser baseado nos princípios da integralidade necessita de revisão das práticas científicas aplicadas na rotina das unidades de saúde, buscando ofertar um ambiente de trabalho menos estressante e que possa valorizar tanto usuário quanto o profissional de enfermagem (ANTUNES P, et al., 2018).

A assistência humanizada deve buscar atender as necessidades e integrais e as humanas básicas dos indivíduos. É atribuição do enfermeiro realizar o planejamento do cuidado, auxiliando o paciente a identificar os seus direitos e deveres buscando sempre tem uma visão holística com o objetivo de assegurar atendimento a para manutenção da qualidade de vida do paciente. Uma das formas de humanização e cuidado é aplicação da Sistematização da Assistência de Enfermagem (SAE) que se constitui como um processo sistemático e organizado para nortear o cuidado fundamentado na prática científica (SANTOS ABV, et al., 2018).

O enfermeiro deve ter o devido reconhecimento acerca do seu trabalho, principalmente, para aqueles que atuam na área da classificação de risco, diante de sua complexidade, bem como, pela responsabilidade teórico-prática que Ihe é imposta. Em meio a todos os desafios e complexidades, ele ainda assim consegue assegurar a garantia do direito à assistência universal, integral e humanizada aos seus usuários, visto que, será o primeiro integrante da equipe multiprofissional a ter contato com o público (SOARES ACL, et al ., 2018). Assim, cabe a este profissional como líder da equipe de enfermagem possuir uma postura ética e seu atendimento e condução do trabalho baseado na humanização. Para a efetivação da humanização na classificação de risco o enfermeiro deve propor ações de educação continuada para a equipe, a fim de garantir a qualidade do atendimento e a melhora contínua da assistência prestada (FREITAS FFB, 2015).

A humanização se torna um desafio para o enfermeiro na classificação de risco, visto que a alta demanda de atendimento e as grandes filas comprometem esse processo entre a equipe e usuários. Além disso, a falta de leitos, a escassez dos recursos humanos, a sobrecarga de trabalho e um fluxo desordenado. Analisando por essa perspectiva talvez esteja aí a maior dificuldade para a humanização no atendimento, tendo em vista que o profissional também é um ser humano com necessidades e que essas precisam ser trabalhadas e supridas por gestores de saúde, para que isso reflita de forma positiva em seu atendimento com pacientes e usuários que buscam o serviço de saúde (SOUZA JA, et al., 2017 ).

\section{CONSIDERAÇÕES FINAIS}

É importante considerar que para praticar a humanização não se deve esperar que o ambiente se torne mais propício a isto, tendo em vista, que principalmente nos serviços públicos de saúde, existe uma carência de recursos materiais. A humanização dessa forma deve ocorrer em qualquer espaço e a qualquer momento, pois vai além de apenas um atendimento clínico realizado pelo profissional. A classificação de risco é uma ferramenta importante dentro dos serviços de urgência e emergência, que possuem como finalidade organizá-los de forma a praticar a escuta qualificada e priorizar os casos mais graves. Além disso, é considerada como um meio de promover a humanização nos serviços de urgência e emergência, principalmente através da escuta qualificada, observando não apenas aspectos da doença como também 
incluir os psicossociais. Dentro desse contexto, o enfermeiro geralmente é o profissional responsável por essa classificação e sua assistência deve ser baseada na humanização podendo ser alcançada através da escuta qualificada, prestando uma assistência segura, resolutiva e que considere esse paciente na sua integralidade.

\section{REFERÊNCIAS}

1. ANTUNES $P$, et al. A importância do atendimento humanizado nos serviços de urgência e emergência: uma revisão de literatura. Revista Científica FacMais, 2018; 12 (1): 1-17.

2. ASSIS LRS, et al. Atendimento humanizado no serviço de urgência e emergência: uma revisão sistemática. Revista Uningá, 2016; 25 (1): 131-35.

3. BARTEL TE, et al. Dialogando sobre serviços de saúde a partir da implantação do acolhimento com avaliação e classificação de risco: relato de experiência. Revista Baiana de Saúde Pública, 2015; 39 (1): 164-73.

4. BRASIL. Ministério da Saúde. Política Nacional de Humanização - HumanizaSUS: 1 ago. 2020.

5. CALEGARI RC, et al. Humanização da assistência à saúde na percepção de enfermeiros e médicos de um hospital privado. Rev Esc Enferm USP, 2015; 49(2): 42-47.

6. COFEN. Resolução COFEN no 423/2012. Normatiza no Âmbito do Sistema Cofen/Conselhos Regionais de Enfermagem, a Participação do Enfermeiro na Atividade de Classificação de Riscos, 2012.

7. FREITAS FFB, et al. O papel do enfermeiro no serviço de Acolhimento e classificação de risco no setor de urgência e emergência. Revista Interdisciplinar em Saúde, 2015; 2 (3): 314-33.

8. MENEZES ML, SANTOS LRCS. Humanização na atenção primária à saúde: um olhar sobre o trabalhador da saúde. Rev. Saúde.Com, 2017; 13 (1): 786-96.

9. NETO OC, et al. A Atuação do Enfermeiro no Sistema de Acolhimento e Classificação de Risco nos Serviços de Saúde. J Health Sci; 2018; 20 (4): 295-302.

10. OLIVEIRA JLC, et al. Acolhimento com classificação de risco: percepção de usuários de uma unidade de pronto atendimento. Texto Contexto Enferm, 2017; (26) 1: 1-8.

11. PAGLIOTO LF, et al. Classificação de Risco em uma unidade de urgência e emergência do interior Paulista. CuidArt enfermagem,2016; 10 (2): 148-55.

12. PAULA CFB, et al. Humanização da assistência: acolhimento e triagem na classificação de risco. Rev enferm UFPE on line. 2019; 13 (4): 997-1005.

13. PRUDÊNCIO CPG, et al. Percepção de enfermeira(o)s sobre acolhimento com classificação de risco no serviço de pronto atendimento. Revista Baiana de Enfermagem, 2016; 30 (2): 1-10.

14. ROCHA TRA, PINTO FO. A humanização na assistência de enfermagem em unidades de urgência e emergência. Revista Científica Interdisciplinar, 2016; 3 (4):146-55.

15. SANTOS ABV, et al. A humanização no cuidado aos pacientes vítimas de queimaduras. REFACI, Brasília, $2018 ; 1$ (1): 1-9.

16. SANTOS TTSM. Humanização em unidades de urgência e emergência, PE. Trabalho de Conclusão de Curso (Pósgraduação em urgência e emergência). Faculdade/Universidade Anhanguera Educacional, 2017, 29 p.

17. SOARES ACL, et al. Acolhimento com classificação de risco: atuação do enfermeiro na urgência e emergência. Revista Recien, 2018; 8 (22): 22-33.

18. SOUSA KHJF, et al. Humanização nos serviços de urgência e emergência: contribuições para o cuidado de enfermagem. Revista Gaúcha de Enfermagem, 2018; 40 (1): 1-10.

19. FILHO LAMS. Revisão sistemática do Sistema de Triagem de Manchester na estratificação de risco, BA. Trabalho de Conclusão de Curso (Bacharelado em Enfermagem). Universidade Federal da Bahia, UFB Salvador; 2013.

20. SOUZA JA, et al. Stresse em serviço de urgência e os desafios para enfermeiros brasileiros e portugueses. Revista de Enfermagem Referência, 2017; 4 (12): 107-16. 